\title{
A CLASS OF ONE-PARAMETER NONLINEAR SEMIGROUPS WITH DIFFERENTIABLE APPROXIMATING SEMIGROUPS
}

\author{
G. EDGAR PARKER
}

\begin{abstract}
Suppose that $T$ is a strongly continuous semigroup of transformations on a subset $C$ of a Banach space $X$. For $\delta>0$, consider $U_{\delta}(t)=$ $\left\{\left(\delta^{-1} \int_{0}^{\delta} g_{x}, \delta^{-1} \int_{0}^{\delta} g_{T(t) x}\right): x \in C\right\}$ where $g_{x}$ denotes the trajectory of $T$ from $x$. The class $H$ of semigroups for which $U_{\delta}(t)$ is a function for $\delta>0$ and $t>0$ contains all strongly continuous linear semigroups and Webb's nonlinear nonexpansive example with no dense set of differentiability. If $T \in H, U_{\delta}=\left\{\left(t, U_{\delta}(t)\right): t \geqslant 0\right\}$ is a semigroup on $\left\{\delta^{-1} \int_{0}^{\delta} g_{x}: x \in C\right\}$ with continuously differentiable trajectories. Also, as $\left\{\delta_{n}\right\}_{n=1}^{\infty}$ converges to 0 , the trajectories of $\left\{U_{\delta_{n}}\right\}_{n=1}^{\infty}$ uniformly approximate the trajectories of $T$.
\end{abstract}

1. Introduction. This paper deals with the construction of differentiable semigroups which uniformly approximate a given strongly continuous semigroup. In the Hille-Yosida theory for strongly continuous semigroups of continuous linear transformations, points of the form $\delta^{-1} \int_{0}^{\delta} g_{x}, \delta>0$, are known to have the property that if $p$ is one of them, then $g_{p}$, the trajectory of the semigroup from $p$, is differentiable. Thus linear semigroups have differentiable trajectories from a dense set of points. That this dense differentiability need not occur in nonlinear semigroups on Banach spaces was first shown in an example due to Webb (see [4]). Even lacking differentiability, however, Brezis and Pazy [1] and Crandall and Liggett [2] have shown that if a semigroup $T$ of local type is given by an exponential formula

$$
T(t) x=\lim _{n \rightarrow \infty}(I+(t / n) A)^{-n} x,
$$

where $A$ is computed from $(I-(t / \delta)(T(\delta)-I))^{-1}$, then the trajectories of $T$ are uniformly approximated by the semigroups whose trajectories are solutions to

$$
y^{\prime}(t)=\delta^{-1}(T(\delta)-I)(y(t)) .
$$

The theorem to follow gives a different approximation technique not dependent upon the existence of an exponential generator. Define, for a given strongly continuous semigroup $T$ on a subset $C$ of a Banach space $X$, a relation $U_{\delta}(t), \delta>0, t \geqslant 0$, so that $U_{\delta}(t)\left(\delta^{-1} \int_{0}^{\delta} g_{x}\right)=\delta^{-1} \int_{0}^{\delta} g_{T(t) x}$ for each point $x$ of $C$. The collection $H$ of semigroups for which $U_{\delta}(t)$ is a function for all positive $\delta$ and nonnegative $t$ includes Webb's example and all strongly

Presented to the Society, November 20, 1976; received by the editors December 15, 1976.

AMS (MOS) subject classifications (1970). Primary 47H15; Secondary 20M20.

Key words and phrases. Nonlinear semigroup of transformations, Hille-Yosida theory, resolvent formula, trajectory, strongly continuous, exponential generator.

(c) American Mathematical Society 1977 
continuous linear semigroups. Note that $T$ is in class $H$ if $x \neq y$ implies $\int_{0}^{\delta} g_{x} \neq \int_{0}^{\delta} g_{y}$. Given $T$ in $H, \delta>0$, and $A_{\delta}=\left\{\left(\delta^{-1} \int_{0}^{\delta} g_{x}, \delta^{-1}(T(\delta)-I) x\right): x\right.$ $\in C\}, \quad\left\{\left(t, U_{\delta}(t)\right): t \geqslant 0\right\}$ is a semigroup on $\left\{\delta^{-1} \int_{0}^{\delta} g_{x}: x \in C\right\}$ with differentiable trajectories satisfying $y^{\prime}(t)=A_{\delta}(y(t))$.

Conditions for a semigroup on a subset of $C_{[0,1]}$ are given which guarantee its inclusion in $H$ and examples are presented which may indicate what type of semigroups may be expected to belong to $H$.

\section{Definitions and theorems.}

Notation. In the following definitions and theorems, $X$ denotes a Banach space, and $C$ denotes a subset of $X$. $I$ denotes the identity function on a given subset of $X$ and $j$ denotes the identity function on the numbers.

Definition. The statement that $T$ is a semigroup on $C$ means that (i) $T:[0, \infty) \rightarrow\{f: f: C \rightarrow C\}$, (ii) $T(0)=I$, and (iii) if $t_{1}$ and $t_{2}$ are nonnegative numbers

$$
T\left(t_{1}+t_{2}\right)=T\left(t_{1}\right)\left(T\left(t_{2}\right)\right) \text {. }
$$

Definition. The statement that $g_{x}$ is a trajectory of the semigroup $T$ on $C$ from the point $x$ means that $g_{x}=\{(t, T(t) x): t \in[0, \infty)\}$.

Definition. The statement that the semigroup $T$ is strongly continuous means that each trajectory of $T$ is continuous.

Definition. Suppose that $T$ is a strongly continuous semigroup on $C$. The statement that $T$ is in class $H$ means that if $\delta>0$ and $t \geqslant 0$, then $\left\{\left(\delta^{-1} \int_{0}^{\delta} g_{x}, \delta^{-1} \int_{0}^{\delta} g_{T(t) x}\right): x \in C\right\}$ is a function, that is, is single-valued.

THEOREM 1. Suppose that $T$ is a strongly continuous semigroup on $C$ and $T$ is in class $H$. For $\delta>0$, define $C_{\delta}=\left\{\delta^{-1} \int_{0}^{\delta} g_{x}: x \in C\right\}$. For $t \geqslant 0$, define $U_{\delta}(t): C_{\delta} \rightarrow C_{\delta}$ by

$$
U_{\delta}(t)\left(\delta^{-1} \int_{0}^{\delta} g_{x}\right)=\delta^{-1} \int_{0}^{\delta} g_{T(t) x}
$$

and $A_{\delta}: C_{\delta} \rightarrow X$ by

$$
A_{\delta}\left(\delta^{-1} \int_{0}^{\delta} g_{x}\right)=\delta^{-1}((T(\delta)-I) x) .
$$

Then (i) $\left\{\left(t, U_{\delta}(t)\right): t \geqslant 0\right\}$ is a semigroup on $C_{\delta}$ with continuously differentiable trajectories satisfying $y^{\prime}(t)=A_{\delta}(y(t))$, and (ii) if $x \in C$ and $\left\{\delta_{n}\right\}_{n=1}^{\infty}$ is a sequence of positive numbers converging to 0 , then if $y_{n}$ is the trajectory of $U_{\delta_{n}}$ from $\delta_{n}^{-1} \int_{0}^{\delta_{n}} b_{x},\left\{y_{n}\right\}_{n=1}^{\infty}$ converges uniformly to $g_{x}$ on every interval in $[0, \infty)$.

THEOREM 2. Suppose that $T$ is a strongly continuous semigroup on the subset $C$ of $C_{[0,1]}$ so that if $f$ and $g$ are points of $C$ and $f(x)>g(x)$, then

$$
[T(t) f](x) \geqslant[T(t) g](x)
$$

for each number $t$ in $[0, \infty)$.

Then $T \in H$. 


\section{Proofs of the theorems.}

Theorem 1. Denote by $S$ the strongly continuous semigroup of nonexpansive linear transformations on the Banach space $Y$ of bounded uniformly continuous functions from $[0, \infty)$ into $X$ (with lub norm) whose action at a point $f$ of $Y$ is $S(t) f=f(j+t)$. By definition of $j,[S(t) f](\delta)=f(j+t)(\delta)$ $=f(\delta+t)$. (The use of a linear semigroup to study nonlinear semigroups was suggested by the work of Neuberger [3].) From the Hille-Yosida theory of strongly continuous linear semigroups, if $F_{f}$ is the trajectory of $S$ from $f \in Y$ and $\delta>0$, the trajectory from $\delta^{-1} \int_{0}^{\delta} F_{f}$ is differentiable. Furthermore, if $A$ is defined by

$$
A\left(\delta^{-1} \int_{0}^{\delta} F_{f}\right)=F_{\delta^{-1} \int_{0}^{\delta} F_{f}}^{\prime}(0),
$$

then the integral equation

$$
(S(t)-I) \int_{0}^{r} F_{f}=(S(r)-I) \int_{0}^{t} F_{f}
$$

implies that $A\left(\delta^{-1} \int_{0}^{\delta} F_{f}\right)=\delta^{-1}(S(\delta)-I) f$.

Let $\delta>0$ and $C_{\delta}$ and $U_{\delta}(t)$ be as in the hypothesis. Then $U_{\delta}(t)$ is a function from $C_{\delta}$ to $C_{\delta}$ by assumption. $U_{\delta}(0)=\left\{\left(\delta^{-1} \int_{0}^{\delta} g_{x}, \delta^{-1} \int_{0}^{\delta} g_{T(0) x}\right): x \in\right.$ C $\}=I$.

$$
\begin{aligned}
U_{\delta}(t+s)\left(\delta^{-1} \int_{0}^{\delta} g_{x}\right) & =\delta^{-1} \int_{0}^{\delta} g_{T(t+s) x}=\delta^{-1} \int_{0}^{\delta} g_{T(t) T(s) x} \\
& =U_{\delta}(t)\left(U_{\delta}(s)\left(\delta^{-1} \int_{0}^{\delta} g_{x}\right)\right)
\end{aligned}
$$

Thus $U_{\delta}$ is a semigroup on $C_{\delta}$.

The strong continuity of $U_{\delta}$ is demonstrated by showing that a given trajectory is continuously differentiable. Suppose $t \geqslant 0, h_{x}$ is the trajectory of $U_{\delta}$ from $\delta^{-1} \int_{0}^{\delta} g_{x}$, and $f$ is an element of $Y$ which agrees with $g_{x}$ on $[0, \delta+t]$. Riemann sum approximations to $\delta^{-1} \int_{0}^{\delta} F_{f}$ converge uniformly to the function $\delta^{-1} \int_{0}^{\delta} F_{f}$ and thus converge pointwise as well. Thus

$$
\begin{aligned}
{\left[\lim _{n \rightarrow \infty} \sum_{k=1}^{n} n^{-1} f(j+(k \delta / n))\right](t) } & =\lim _{n \rightarrow \infty} \sum_{k=1}^{n} n^{-1} f(t+(k \delta / n)) \\
& =\left[\delta^{-1} \int_{0}^{\delta} F_{f}\right](t) .
\end{aligned}
$$

Since $f$ agrees with $g_{x}$ on $[0, \delta+t]$,

$$
\begin{aligned}
\sum_{k=1}^{n} n^{-1} f(t+(k \delta / n)) & =\sum_{k=1}^{n} n^{-1} g_{x}(t+(k \delta / n)) \\
& =\sum_{k=1}^{n_{n-1}} n^{-1} T(t+(k \delta / n)) x \\
& =\sum_{k=1}^{n} n^{-1} T((k \delta) / n) T(t) x
\end{aligned}
$$


Thus $\left[\delta^{-1} \int_{0}^{\delta} F_{f}\right](t)=\delta^{-1} \int_{0}^{\delta} g_{T(t) x}=U_{\delta}(t)\left(\delta^{-1} \int_{0}^{\delta} g_{x}\right)=h_{x}(t)$ and $h_{x}$ on any interval is a domain element of $A$ on that interval and is therefore continuously differentiable. Furthermore,

$$
\begin{aligned}
h_{x}^{\prime}(t) & =A\left(\delta^{-1} \int_{0}^{\delta} F_{f}\right)(t)=\left[\delta^{-1}(S(\delta)-I) f\right](t) \\
& =\delta^{-1}(f(j+\delta)-f)(t)=\delta^{-1}\left(g_{x}(t+\delta)-g_{x}(t)\right) \\
& =\delta^{-1}(T(\delta)-I)(T(t) x) .
\end{aligned}
$$

Therefore, $h_{x}^{\prime}(t)=A_{\delta}\left(h_{x}(t)\right)$.

Suppose $x \in C$ and $\left\{\delta_{n}\right\}_{n=1}^{\infty}$ is a sequence of positive numbers converging to 0 . Then $\left\{\delta_{n}^{-1} \int_{0}^{\delta_{n}} F_{f}\right\}_{n=1}^{\infty}$ converges uniformly to $f$. Since $f$ agrees with $g_{x}$ on $[0, t]$, the trajectories of $U_{\delta_{n}}$ from $\delta_{n}^{-1} \int_{0}^{\delta_{n}} g_{x}$ which are the restrictions of $\delta_{n}^{-1} \int_{0}^{\delta_{n}} F_{f}$ to $[0, t]$ converge uniformly to $g_{x}$ on $[0, t]$.

Proof OF THEOREM 2.

Lemma. Suppose that $T$ is a strongly continuous semigroup on the subset $C$ of $C_{[0,1]}$. For $f \in C$, define a collection of functions $\left\{f^{x}\right\}_{x \in[0,1]}$ from $[0, \infty) \rightarrow \mathbf{R}$ by $f^{x}(t)=[T(t) f](x)$. Suppose further that if $\delta>0$ and $f$ and $h$ are points of $C$, then there is $x \in[0,1]$ so that $\delta^{-1} \int_{0}^{\delta} f^{x} \neq \delta^{-1} \int_{0}^{\delta} h^{x}$. Then $T \in H$.

Suppose the hypothesis holds and $f$ and $h$ are in $C$. Then

$$
\left[\delta^{-1} \int_{0}^{\delta} g_{f}\right](x)=\delta^{-1} \int_{0}^{\delta} f^{x} \text { and }\left[\delta^{-1} \int_{0}^{\delta} g_{h}\right](x)=\delta^{-1} \int_{0}^{\delta} h^{x} \text {. }
$$

Since $f$ and $h$ are different, there is a number $x$ where these integrals are different. Thus $f \neq h$ implies $\delta^{-1} \int_{0}^{\delta} g_{f} \neq \delta^{-1} \int_{0}^{\delta} g_{h}$ and $U_{\delta}(t)$ must be a function for any number $t \in[0, \infty)$. Thus $T \in H$.

Consider $T$ as in the hypothesis to Theorem 2 and suppose $f \neq h$. Then there is $x \in[0,1]$ so that $f(x) \neq h(x)$. Suppose $f(x)>h(x)$. Then if $t \geqslant 0$, $[T(t) f](x) \geqslant[T(t) h](x)$. But $T$ is strongly continuous, so

$$
\lim _{t \rightarrow 0}[T(t) f](x)=f(x) \text { and } \lim _{t \rightarrow 0}[T(t) h](x)=h(x) .
$$

Thus there is $\varepsilon>0$ so that if $t \in[0, \varepsilon],[T(t) f](x)>[T(t) h](x)$ and $\delta^{-1} \int_{0}^{\delta} f^{x}$ $>\delta^{-1} \int_{0}^{\delta} h^{x}$. By the Lemma, $T \in H$.

4. Examples. (A) (G. F. WebB, SEe [4]). Let $X=C=C_{[0,1]}$. If $f \in C_{[0,1]}$, and $x \in[0,1]$, define

$$
[T(t) f](x)= \begin{cases}t+f(x) & \text { if } f(\dot{x}) \geqslant 0, \\ t+(1 / 2) f(x) & \text { if } f(x)<0 \text { and } t+(1 / 2) f(x) \geqslant 0 \\ 2 t+f(x) & \text { if } t+(1 / 2) f(x)<0 .\end{cases}
$$

(B) (Crandall and Liggett [2]). Let $X=C_{[0,1]}$ and let $C$ be the set to which $f \in X$ belongs provided that if $x \in[0,1], 0 \leqslant f(x) \leqslant x$. Define

$$
[T(t) f](x)=\min \{t+f(x), x\} \text {. }
$$


(C) Let $X=C=C_{[0,1]}$. If $f \in X$, denote by $f^{*}$ the maximum value of the function $f$ and by $f_{*}$ the minimum value of $f$. Define

$$
[T(t) f](x)= \begin{cases}\min \left\{t+f(x), f^{*}\right\} & \text { if there is } x \text { in }[0,1] \\ f_{*}+t & \text { so that } t+f(x) \leqslant f^{*},\end{cases}
$$

(A) and (B) are examples having the "order" property in Theorem 2. Example (C) fails to have this order property but satisfies the hypothesis to the Lemma to Theorem 2.

5. Concluding remarks. The approximation theorem in this paper seems to be different from those using resolvents. In general, the trajectories of $U_{\delta}$ are differentiable at all numbers. Furthermore, $A_{\delta}$ might be viewed as a perturbation of $\delta^{-1}(T(\delta)-I)$ in the sense of a shift in the domain rather than the range. The full extent to which semigroups given by resolvent formulas and those of class $H$ are related is not known to the author. Two questions seem to be of special interest.

(i) Do there exist conditions on $C$ which imply that a semigroup of nonexpansive transformations on $C$ be in $H$ ?

(ii) Does there exist a semigroup of nonexpansive transformations given by a resolvent formula and defined on the closure of a convex open set which is not in $H$ ?

\section{REFERENCES}

1. H. Brezis and A. Pazy, Convergence and approximation of semigroups of nonlinear operators in Banach spaces, J. Functional Analysis 9 (1972), 63-74.

2. M. G. Crandall and T. M. Liggett, Generation of semigroups of nonlinear transformations on general Banach spaces, Amer. J. Math. 93 (1971), 265-298.

3. J. W. Neuberger, Lie generators for strongly continuous equiuniformly continuous one parameter semigroups on a metric space, Indiana Univ. Math. J. 21 (1972), 961-971.

4. __, Quasi-analyticity and semigroups, Bull. Amer. Math. Soc. 78 (1972), 909-922.

Department of Mathematics, Emory University, Atlanta, Georgia 30322

Current address: Department of Mathematics, Pan American University, Edinburg, Texas 78539 